\title{
WILDTIERE IN HISTORISCHEN GÄRTEN
}

\begin{abstract}
Wildtiere spielen in historischen Gärten heute sehr unterschiedliche Rollen. Sie waren oftmals Grund für deren Etablierung (Tiergärten) oder Teil der Installation (Landschaftsgärten). Heute besiedeln Wildtiere die Gärten meist selbstständig und ohne menschliches Zutun. Ihre Anwesenheit kann, je nach Sichtweise, als positiv (Bereicherung des Gartenaspektes, Artenschutz) oder als ausgesprochen negativ (Schäden, insbesondere an Vegetation und Gebäuden, Verdrängung heimischer Arten durch invasive Neozoen) empfunden werden. Gelegentlich, wenn etwa gefährdete oder zumindest geschützte Arten wie Biber oder Fischotter große Schäden verursachen, lässt sich selbst diese Differenzierung nicht mehr klar treffen. Der vorliegende Beitrag zeigt Beispiele für die Rolle unterschiedlicher Tierarten in historischen Gärten und diskutiert mögliche Managementansätze, um in ihnen bestimmte Arten unter den spezifischen Bedingungen des urbanen Lebensraumes mit sehr heterogenen Ansichten und Einstellungen der Bevölkerung entweder zu fördern, zu tolerieren oder aus den Gärten fernzuhalten.
\end{abstract}

Nowadays wildlife plays greatly differing roles in historic gardens. It was often the reason for their establishment ("deer parks") or part of the installation (landscape gardens). Today wildlife mostly populates gardens independently and without any human input. Depending on the stance taken, their presence can be seen as positive (enhancement of the garden aspect, species conservation) or as extremely negative (damage, particularly to vegetation and buildings, displacement of indigenous species by invasive neozoa). Sometimes when endangered or at least protected species, such as beavers or otters, cause major damage, even this differentiation may no longer be clear. This article gives examples of the role of different animal species in historic gardens. It discusses possible management approaches to promote, toler-

Im Text werden Maskulinum und Femininum verwendet, wenn es um Personen geht. Gemeint sind grundsätzlich alle Menschen, gleich welcher Geschlechtsidentität sie sich zugehörig fühlen. 
ate or keep out certain species in the gardens under the special conditions of the urban habitat that comes with very heterogeneous views and attitudes of the human population.

\section{Einleitung}

Tiere (Wildtiere und Haustiere) spielten in historischen Gärten regelmäßig eine wichtige Rolle. Allerdings war und ist sie je nach Epoche und auch Sichtweise sehr unterschiedlich und umfasst ein weites Spektrum der Wahrnehmung. So waren Wildtiere nicht selten ein zentraler Bestandteil des Konzeptes eines Gartens. Viele Arten werden andererseits heute sei es aufgrund ihrer Fraßeinwirkungen, sei es durch andere unerwünschte Einflüsse auf die Komposition des Gartens oder auf die Besucher - als schädlich angesehen und mit einer negativen Wahrnehmung verknüpft. Diese Tiere sind Ursachen für entsprechende Konflikte. Vorliegender Beitrag zeigt, dass historische Gärten geeignet sind, das gesamte Spektrum des Mensch-Tier-Verhältnisses in Gesellschaften der Vergangenheit und Gegenwart wie unter einem Vergrößerungsglas abzubilden (vgl. Herzog 2019).

\section{Wildtiere in urbanen Räumen}

Weltweit lebt ein zunehmender Anteil der menschlichen Bevölkerung in Städten. So stieg der Anteil urbaner Bevölkerung stetig von rund $30 \%$ in den $1950 \mathrm{er}$ Jahren auf über $50 \%$ in den frühen 2000er Jahren. Für 2050 wird ein Anteil der Stadtbevölkerung von drei Vierteln an der Weltbevölkerung insgesamt prognostiziert. Da dieses Phänomen allerdings mit einer absolut rasch steigenden Bevölkerungszahl einhergeht, kommt es weltweit gleichzeitig auch zu einem Bevölkerungswachstum in den meisten ländlichen Lebensräumen, die dadurch ihrerseits zunehmend urban überformt werden. Letzteres finden wir allerdings in Mitteleuropa weniger ausgeprägt. Hier gibt es tatsächlich Landschaften mit deutlichem Bevölkerungsschwund, woraus sich ganz eigene Herausforderungen ergeben.

Städte und allgemein urbane Lebensräume haben aus Sicht von Wildtieren zwei Gesichter: Mit der Verdrängung natürlicher und naturnaher Lebensräume durch Siedlungen, Industrie und die entsprechende Infrastruktur ist zunächst ein Verlust an Arten verbunden. Urbanisation wird (neben der industrialisierten Landwirtschaft) weltweit als eine der wichtigsten Ursachen für den Verlust von Tier- und Pflanzenarten angesehen (McKinney 2002). Die durch Urbanisation verursachten Landschaftsveränderungen werden auch deshalb als besonders gravierend angesehen, weil sie kaum reversibel sind (Stein et al. 2000).

Störfaktoren des urbanen Umfeldes für Wildtiere sind insbesondere

- die permanente Präsenz des Menschen (vgl. Ditchkoff et al. 2006; Partecke et al. 2006).

- Heimtiere, insbesondere Hunde und Hauskatzen. Letztere sind aufgrund ihrer permanenten und ubiquitären Anwesenheit eine ernsthafte Bedrohung für zahlreiche 
Singvogel- und Reptilienarten (vgl. Hackländer et al. 2014; McDonald et al. 2015). Freilaufende Hunde wiederum sind weniger ein Artenschutz-, sondern ein Tierschutzproblem (vgl. Herzog 2019).

- »Ersatz« lokaler und lokal angepasster Arten durch heimische oder auch angesiedelte anpassungsfähige und konkurrenzstarke Arten, sogenannte »Allerweltsarten « (siehe z.B. McKinney 2006).

- Lichtverschmutzung durch Städte, ein Problem vor allem für nachtaktive und solche Arten, die von der Photoperiode abhängig sind (vgl. Longcore/Rich 2004).

- Beeinträchtigungen durch Lärm, insbesondere für Arten mit akustischer Dominanz (vgl. z.B. Brumm 2004; Dominoni et al. 2016).

Zugleich haben Städte auch zahlreiche positive Wirkungen. Sie geben Pflanzen und Tieren Lebensräume einschließlich spezifischer Nischenlebensräume wie beispielsweise Ruderalflächen und stellen, unter anderem durch das Vorhandensein großer urbaner Grünflächen, auch Refugien für verschiedene Arten dar. In diesem Zusammenhang müssen wir anerkennen, dass zahlreiche Tierarten durch (genetische) Anpassungsvorgänge oder durch Habituation, also letztlich Lernvorgänge, die je nach Tierart auch als Tradition innerhalb von Gruppen über Generationen weitergegeben werden, anthropogene und somit auch urbane Lebensräume für sich entdeckt haben. Dies führt so weit, dass die klassischen Kategorien der »Kulturfolger« und »Kulturflüchter« heute kaum noch Gültigkeit haben (Herzog 2019). Vielmehr erkennen wir, dass zahlreiche Arten, die ursprünglich als extreme Kulturflüchter galten, heute die Nähe des Menschen suchen und von bestimmten ökologischen Gegebenheiten urbaner Lebensräume profitieren. Typische Beispiele hierfür sind Wanderfalke (Falco peregrinus, s.u.), Uhu (Bubo bubo), Graureiher (Ardea cinerea), Wolf (Canis lupus) und - ganz aktuell - Seeadler (Haliaeetus albicilla). Letzterer tritt zwar noch nicht im urbanen Umfeld auf, aber Seeadler als traditionelle Bewohner weiter, ungestörter Wälder mit großen Gewässern finden sich zunehmend nach Beute suchend etwa entlang von Verkehrswegen. Dies zeigt, dass solche evolutiven Anpassungen von ehemals scheuen Tieren auch rezent stattfinden.

Die Attraktivität urbaner Lebensräume für Wildtiere kann verschiedene Gründe haben. Zunächst fehlt innerhalb von Städten der menschliche Jagddruck. Dies trifft in Mitteleuropa in erster Linie für diejenigen Arten zu, die einer regulären jagdlichen Nutzung unterliegen. Ein weiterer wichtiger Aspekt ist die Tatsache, dass Städte für viele Arten hinreichend Nahrung bieten. Geist (2007) sieht hierin bzw. in der daraus resultierenden Habituation ein zentrales Problem. Dies trifft ganz besonders auf Nahrungsgeneralisten wie den Fuchs (Vulpes vulpes) oder das Schwarzwild (Sus scrofa), aber durchaus auch auf Spezialisten wie das Reh (Capreolus capreolus) zu. Dabei sind es nicht nur die menschlichen Pflanzungen oder Abfälle, die für zahlreiche Tierarten attraktiv sind, man denke hier beispielsweise an die Krähen (Corvus corone) oder auch an den Fuchs oder Neozoen (also aus anderen biogeographischen Regionen eingeschleppte Arten) wie den Waschbären (Procyon lotor). Darüber hinaus profitieren beispielsweise Prädatoren auch von der Anwesenheit zahlreicher Beutetierarten, das Beispiel des Wanderfalken wurde bereits erwähnt. 
Steinmarder (Martes foina), Fuchs und Iltis (Mustela putorius) profitieren nicht nur von zahlreichen Vogelarten, die in der Stadt leben, sondern auch von den in hoher Dichte vorhandenen Ratten und Mäusen. Gerade die Populationsdichte der Wanderratte (Rattus norvegicus) dürfte künftig deutlich zunehmen, da die in Mitteleuropa derzeit forcierte Trennung von Regen- und Schmutzwasserkanälen die Regulation der Wanderratte durch Starkregenereignisse in Zukunft wohl weitgehend verhindert. Die Abwasserkanäle, in denen Ratten typischerweise leben, werden durch diese Maßnahme bei Starkregen seltener überflutet, sodass der ehemals bedeutende Mortalitätsfaktor »Ertrinken bei Starkregen« zunehmend entfällt.

Neben Beutetieren sind es die menschlichen Abfälle, die sich zahlreiche Arten in den Städten zunutze machen. Von Aaskrähen über Ratten, Füchse, Waschbären und, in manchen Teilen der Welt, Wölfe und Braunbären (Ursus arctos) finden wir zahlreiche Profiteure eines Entsorgungssystems, das an vielen Stellen Wildtieren einen guten $\mathrm{Zu}$-gang zu Abfällen verschafft (vgl. z.B. Bateman/Fleming 2012; Plumer et al. 2014).

Ein weiterer Faktor, der die Urbanisation von Wildtieren fördert, ist das tendenziell wärmere lokale Klima in Städten. Im Sommer kommt es durch die Aufheizung von Gebäuden aus Stein bzw. Beton (»künstliche Felslandschaft« mit einer entsprechenden Thermik, die Greifvögeln die Jagd erleichtert) häufig zur stärkeren Erwärmung als im Umland. Ein reduzierter Abfluss der Wärme in der Nacht kann diesen Effekt verstärken. Weiterhin relevant sind die Abwärme durch Verkehr, Heizungen (im Winter) und sonstigen Energiekonsum. Insgesamt führt dies einerseits zu einer verlängerten Vegetationszeit und andererseits zu geringeren Winterverlusten, gerade bei Kleinvögeln.

Klima und Nahrung sind vermutlich die wesentlichen Ursachen dafür, dass bestimmte Vogelarten, die ursprünglich als Zugvögel oder Teilzieher galten, sich nicht selten in Städten zu Standvögeln entwickeln und dort auch überwintern. Ein Beispiel stellt die Kanadagans (Branta canadensis) dar, die regelmäßig in ein und derselben urbanen Region brütet und überwintert (Hope 2000).

Auch eine längere tägliche Aktivität von Singvögeln wird in Städten beobachtet. Die Gesangsaktivität beginnt morgens früher und endet abends später. Bei Wanderfalken wird beobachtet, dass sie auch nachts ihre Jungen füttern (Adams 2016). Ob dies mit der »Lichtverschmutzung « in Städten zusammenhängt, wissen wir nicht, es ist aber zu vermuten, und ob es letztlich für die betroffenen Arten im Einzelfall eher günstige oder ungünstige Folgen hat, bedarf weiterer Untersuchungen. Beim Wanderfalken geht man davon aus, dass es neben einer gewissen Desorientierung der Beutearten (DeCandido/Allen 2006) oder einer Attraktivität des nächtlichen Lichtes für bestimmte Beutearten nicht zuletzt die Kombination aus Beleuchtung und Schatten ist, die ihm die Jagd auch in der Dunkelheit ermöglicht. ${ }^{1}$

Schließlich wechseln sich in Städten kleinräumig unterschiedliche Nutzungsformen der Grundstücke ab, es finden sich unterschiedlich strukturierte Grünflächen, teilweise sind Städte von umfangreichen Gewässersystemen durchzogen, und daneben finden sich 
immer wieder, meist passager, Brachflächen und Baustellen. Diese Vielfalt an Mikrohabitaten ist es, die einer vergleichsweise großen Vielfalt an Arten Lebensraum bietet. Dabei sollte allerdings nicht übersehen werden, dass allein die Artenzahl noch keinen Hinweis auf die Stabilität eines Ökosystems darstellt.

\section{Wildtiere in historischen Gärten}

Historische Gärten bieten innerhalb dieses »Patchworks« an unterschiedlichen, vielfältigen, doch meist klein- und kleinstflächigen Lebensräumen ihrerseits relativ große, relativ stabile, relativ naturnahe und vielfältige (Wasser, Wiese, Gehölze, Gebäude) Ankerlebensräume, deren größten Vorteil gegenüber den anderen urbanen Flächen wohl die langfristige Stabilität darstellt. Während etwa eine Ruderalfläche eines unvollendeten oder brachliegenden Bauvorhabens jederzeit komplett umgestaltet werden kann, während ein Fledermausquartier oder ein Greifvogelhorst leicht durch Baumaßnahmen zerstört werden kann, stellt der besondere Denkmalschutzstatus historischer Gärten einen Stabilitätsfaktor dar. Historische Gärten können aus Sicht von Wildtieren im Grunde als kleine »stabile Inseln« innerhalb der sich stetig verändernden großen Insel »Stadt« interpretiert werden.

Für Gärten, die nicht im urbanen, sondern im suburbanen oder gar ländlichen Umfeld bestehen (Beispiel Gartenreich Wörlitz), gilt dies sinngemäß. Ihre Besonderheit liegt in dem noch intensiveren und unmittelbareren Austausch mit dem Umland. Dadurch verstärken sich positive ebenso wie negative Wirkungen von Wildtieren. In einem ländlichen Umfeld wären beispielsweise Hirscharten, sofern gewünscht, relativ leicht in die Gärten zu integrieren. Ebenso stehen diese Gärten unter besonders intensivem Besiedlungsdruck nicht erwünschter Arten, etwa des Schwarzwildes.

Aus Sicht des Menschen können Tiere in historischen Gärten im Grunde entweder

- erwünscht (weil sie attraktiv sind und/oder ein Teil des jeweiligen Gartenkonzeptes),

- unerwünscht (weil sie spezifische Schäden verursachen oder Risiken bergen),

- nur in einer bestimmten Abundanz oder Verteilung erwünscht (weil dann ihre Attraktivität die Schäden überwiegt),

- indifferent (sie stören nicht, aber es besteht auch kein spezifisches Interesse) sein.

\section{Tiere als erwünschter Bestandteil historischer Gärten}

Zahlreiche Tierarten sind in historischen Gärten ausgesprochen erwünscht und oftmals als ihr integraler Bestandteil sogar von großer Bedeutung. Je nach ihrer individuellen Geschichte gibt es große Unterschiede zwischen den historischen Gärten in Bezug auf die Rolle von Wildtieren, aber auch Haustieren. 


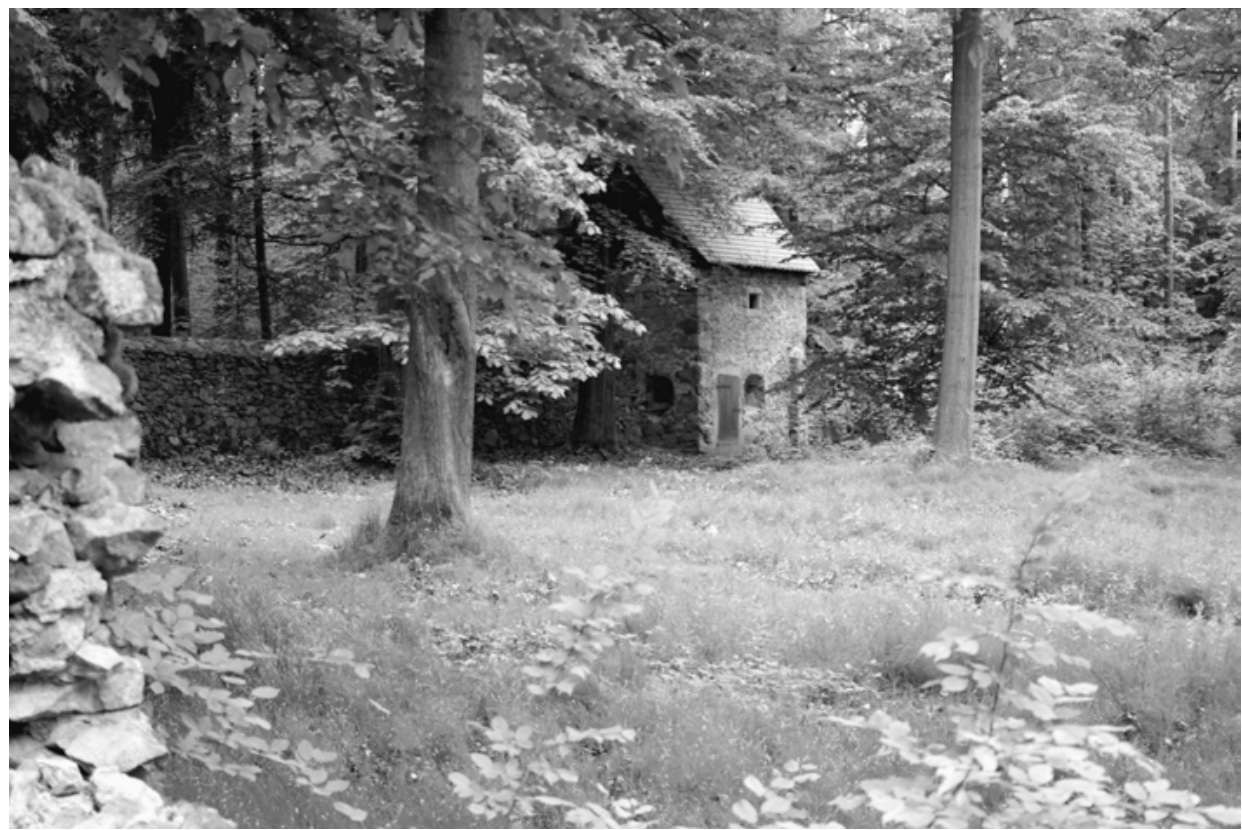

1 Historische Gärten entstanden nicht selten aus sog. Tiergärten, in denen meist große Huftiere für die sog. »höfische Jagd« gehalten wurden. Meist wurden diese später aufgegeben (hier Reste eines sog. Saugartens bei Dresden).

So haben ehemalige Tiergärten einen ganz unmittelbaren Bezug zu Wildtieren. Tiergärten waren und sind Symbol für eine der vier Phasen der Jagd in der Menschheitsgeschichte, die sog. höfische Jagd. Die Anlagen dienten dazu, jagdbares Wild innerhalb eines Areals zu halten. Über gezielte Wegeführungen, z.B. Jagdsterne, waren diese Tiergärten für die höfische Gesellschaft leicht zugänglich.

Heutzutage erinnert meist nichts mehr an die primären Nutzungen (Berliner Tiergarten). Nur selten finden wir Relikte historischer Gebäude in Stadtwäldern (»Saugärten « zum Beispiel in der Dresdner Heide) oder zoologischen Gärten (Wildgehege Moritzburg bei Dresden, Tierpark Schönbrunn bei Wien), die sich aus den ehemaligen Tiergärten entwickelten (Abb. 1). Mit Ausnahme der zoologischen Gärten sind die großen Paarhuferarten heute aus diesen Gärten weitgehend verschwunden. Interessant ist hier die Frage, ob man in der Besiedlung dieser ehemaligen Tiergärten mit Rot-, Schwarz- oder Rehwild nicht die historische Situation in gewisser Weise aufgreifen kann und diese Arten in denjenigen Landschaftsgärten, die eine solche Besiedlung zulassen, gezielt fördern und in die Gartenkonzepte einbeziehen kann.

Anders verhält es sich mit den Landschaftsgärten, die ab dem 18. Jahrhundert im Sinne »idealisierter Landschaften« entstanden. In ihnen spielten sowohl Haus- als auch Wildtiere sehr unterschiedliche Rollen. So können Haustiere Bestandteil der Garteninstallation ent- 
weder aus dem romantischen Motiv einer »idealisierten Kulturlandschaft« oder als Teil eines pädagogischen Konzeptes (Gartenreich Wörlitz) sein. Wildtiere spielen in solchen Landschaftsgärten ebenfalls als Symbol für das Natürliche, gelegentlich auch das »Wilde« eine Rolle. Bedeutsam sind hier einerseits zahlreiche Vogelarten, die für uns Menschen aus unterschiedlichen Gründen grundsätzlich positiv besetzt sind (Wasservögel, Störche, Fasanen, Pfaue), andererseits auch verschiedene (z.T. ebenfalls exotische) Hirscharten, letztere insbesondere in England und Irland. Als besonders problematisch erweisen sich in diesen Gärten heute Arten wie Schwarzwild, Biber, Maulwurf, Wühlmaus und Waschbär. Auch Kaninchen und Rehwild können leicht Probleme bereiten, wenngleich sie oft in das originäre Bild solcher Gärten gehören.

In Volksparks des 18. und 19. Jahrhunderts, oft aus sozialen bzw. sozialromantischen Motiven entstanden, stehen Tiere als Teil der Installation weniger im Vordergrund. Allerdings wurden auch und gerade diese Flächen regelmäßig von Wildtieren besiedelt. Nicht selten bilden solche Anlagen sogar Ausgangspunkte und Trittsteine der Urbanisation von Wildtieren, etwa Singvögeln, Wasservögeln, Rehwild, Feldhase, Fuchs und vielen anderen mehr. Der Schutz vor Prädation und insbesondere Verfolgung durch den Menschen spielt hierbei wohl die entscheidende Rolle. Gleichzeitig entwickeln sich genau diese Tiere hier nicht selten auch zum Problem.

Um in historischen Gärten erwünschte Arten zu fördern, stehen zahlreiche Möglichkeiten der Lebensraumgestaltung zur Verfügung. Klassiker sind dabei Nisthilfen für unterschiedliche Vogelarten (Wanderfalken, Weißstorch (Ciconia ciconia) oder diverse Höhlenbrüter) oder Fledermauskästen. Auch die (Winter-)Fütterung der Singvögel ist ein geeignetes Managementinstrument. Hier geht es um eine Hilfe für Tierarten, denen durch den Menschen wichtige Lebensraumelemente genommen wurden (Berthold 1976). Zunehmend werden allerdings auch Maßnahmen entwickelt, die nicht auf spezifische Arten oder Artengruppen zielen. Kleinräumig war und ist die Anlage von Kleingewässern eine beliebte Maßnahme.

Inwiefern historische Gärten in Maßnahmen zur Vernetzung von Städten mit dem Umland durch begrünte Korridore und Gewässer positiv eingebunden werden können, ist im Einzelfall zu untersuchen. Hierbei ist allerdings zu bedenken, dass derartige "grüne Achsen« auch dazu führen, dass unerwünschte Tierarten leichter den Weg in die Gärten finden.

\section{Konflikte zwischen Menschen und Wildtieren in historischen Gärten}

Die Präsenz von Wildtieren in historischen Gärten führt neben positiven Wirkungen auf die Menschen nämlich auch zu zahlreichen Konflikten. Sie sind meist dadurch gekennzeichnet, dass Tiere Sach- oder Gesundheitsschäden verursachen können oder dass ihre Anwesenheit als störend empfunden wird. Die größte Bedeutung haben in historischen Gärten wohl die Schäden an den wertvollen Außenanlagen, zum Teil allerdings auch an 


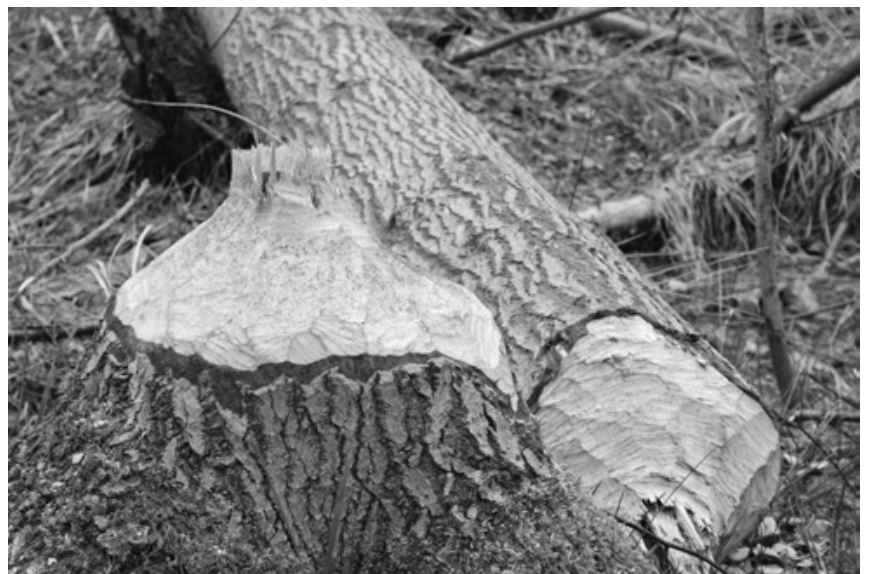

2 Auch unter Schutz stehende Arten wie der Biber (Castor fiber) können in historischen Gärten gravierende Schäden verursachen.

Gebäuden. Insbesondere das Schwarzwild breitet sich in vielen Städten zunehmend von den Randbereichen in die zentral gelegenen Grünflächen und Gärten aus und fällt hier durch umfangreiche Wühl- und Fraßeinwirkungen auf. Auch Kollisionsereignisse mit Fahrzeugen bilden zunehmend ein Problem. Für historische Gärten ist das Wildschwein, ggf. neben dem Biber (Castor fiber), derzeit sicher am problematischsten.

Mehr die Gebäude und weniger die Gartenanlagen selbst stehen bei Waschbär (welcher auch zu den Neozoen gehört, s.u.) und Steinmarder im Vordergrund. Es sind diejenigen Raubsäuger, die nicht selten auf Dachböden leben und hier durch Kinderstuben oder Winterquartiere die menschlichen Belange durch Zerstörungen von Bausubstanz (Dachisolation), Lärm, Verschmutzungen und das Risiko der Krankheitsübertragung (Waschbär) zum Teil erheblich beeinträchtigen. Demgegenüber handelt es sich beim Fuchs, der ebenfalls in Städten in erstaunlich hohen Dichten vorkommt, eher um eine unauffällige Art, die - neben dem Risiko der Übertragung des Fuchsbandwurms - vor allem dort Probleme bereitet, wo Haustiere (Tauben, Ziervögel, Hühner) außerhalb von Gebäuden gehalten werden.

Zunehmend wird, wie erwähnt, auch der Biber in urbanen Räumen beobachtet, wobei das entscheidende Problem derzeit weniger beim Biber selbst, sondern in der Diskrepanz zwischen hohem Schutzstatus und Populationsdynamik der Art liegt. Durch den Biber werden direkte Fraßschäden an Gehölzen verursacht (Abb. 2). Zugleich ist er in der Lage, durch umfangreiche Dammbauaktivitäten Wasserstände von Fließgewässern in seinem Sinne zu beeinflussen. Dies kann zu großflächigen Überflutungen führen. Schließlich kann der Biber auch Uferbefestigungen durch das Graben von Bauen beschädigen. Dies ist auch das Hauptproblem beim Auftreten von Bisam (Ondatra zibethicus) und Nutria (Myocastor coypus).

Weitere Arten verursachen zwar keine substantiellen Zerstörungen in den Gärten, doch beeinträchtigen sie ihre Nutzung. Tauben, insbesondere verwilderte Haustauben 
3 Ehemals scheue Tiere wie der Graureiher (Ardea cinerea) haben sich heute im urbanen Raum gut an den Menschen angepasst.

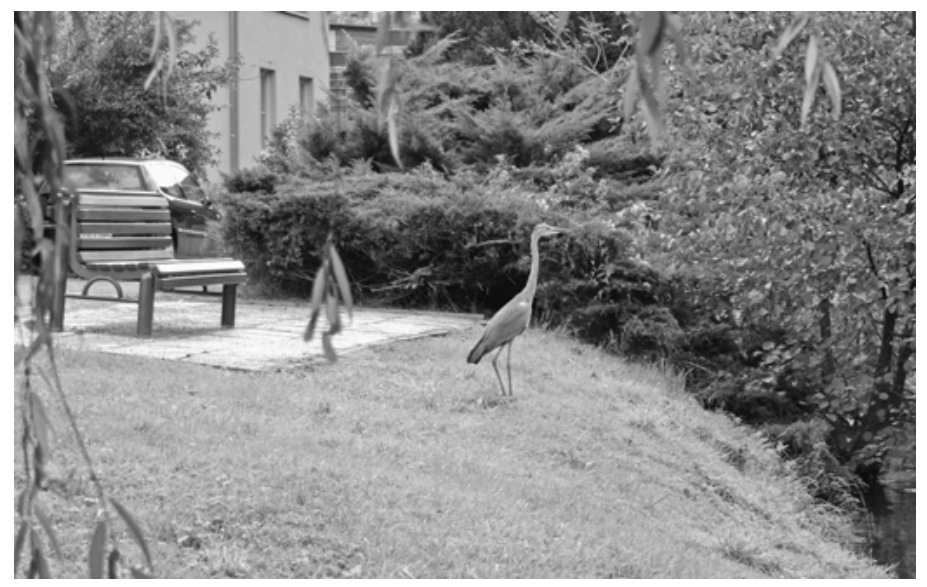

(Felsentauben, Columba livia), Möwen und Krähen, aber auch Wildgänse und gelegentlich Wildenten gehören in diese Kategorie. Bei den genannten Arten sind vor allem Verschmutzungen von Wegen, Plätzen und Gebäuden durch die massiert in großer Zahl auftretenden Tiere und die Übertragung von Krankheitskeimen (z.B. Salmonellen) von Bedeutung.

In den letzten Jahren finden wir auch zunehmend Graureiher in urbanen Regionen, die für Fische in flachen Gewässern ein Problem darstellen können, während Kormorane auch in tieferen Gewässern den Fischbesatz dezimieren können (Abb. 3). Gleiches gilt für den streng geschützten, aber derzeit in deutlicher Zunahme begriffenen Fischotter (Lutra lutra, vgl. auch MacDonald/Mason 1994).

Schließlich gehören auch Neozoen in historischen Gärten oft zu den problematischen Arten. So ging im vergangenen Jahr (z.B. Ring/Jeske 2017) die Meldung durch die Presse, dass im Berliner Tiergarten zunehmend amerikanische Sumpfkrebse auftauchen. Dieses Beispiel zeigt, dass historische Gärten aufgrund ihrer Nähe zum menschlichen Siedlungsraum auch als Eintrittspforten und Trittstein für potentiell invasive Neozoenarten von Bedeutung sind und diesbezüglich eine große Verantwortung tragen.

\section{Umgang mit unerwünschten Tieren: Vergrämen oder verfrachten, bekämpfen oder bejagen?}

In historischen Gärten ist es eine große Herausforderung an ein Wildtiermanagement, diese Probleme zu lösen, ohne gleichzeitig andere Konflikte hervorzurufen.

Die geradezu klassische Situation entsteht, wenn es darum geht, Tiere, die Konflikte verursachen, zu töten. Hier werden im städtischen Umfeld sofort und unabhängig von den zugrundeliegenden Konflikten Gruppen in Erscheinung treten, die sich vehement gegen 
das Töten der Tiere wenden. Somit empfiehlt es sich, im Rahmen der Problemanalyse im Vorfeld bereits möglichst viele gesellschaftliche Gruppen einzubinden. Diese Empfehlung zeigt bereits, dass sich Wildtiermanagement in historischen Gärten nicht unerheblich von den Konzepten im urbanen und suburbanen Raum unterscheidet. Das liegt insbesondere an den soziologischen, kulturellen und ökonomischen Besonderheiten des Umfeldes.

Heute können wir nicht mehr davon ausgehen, dass die Menschen die Abläufe in natürlichen Ökosystemen kennen und dementsprechend Verständnis aufbringen für Eingriffe in Wildtierpopulationen, die diese natürlichen Prozesse bis zu einem gewissen Punkt ersetzen oder auch abbilden sollen. Somit ist es wichtig, genau dieses Verständnis, soweit möglich, herzustellen, bevor Eingriffe in Wildtierbestände im städtischen Umfeld erfolgen. Aus diesem Grunde wird Wildtiermanagement in historischen Gärten immer deutlich vom Einzelfall geprägt sein. Konzepte müssen regelmäßig auf den Einzelfall zugeschnitten werden. Dazu gehört vor dem genannten sozio-kulturellen Hintergrund urbaner Bevölkerungen auch, dass »winning hearts and minds« regelmäßig spezifischen Maßnahmen vorzuschalten ist.

Sofern das Wildtiermanagement also nicht im Rahmen einer Routine erfolgt, beziehungsweise wenn Probleme auftreten, die durch das implementierte Management nicht abgedeckt werden, bietet sich beispielsweise ein professionell (idealerweise durch Wildbiologen mit Moderatorenausbildung) moderierter Workshop zur Analyse des Problems und der möglichen Lösungswege an. Das mag auf den ersten Blick übertrieben erscheinen, ist aber aus Erfahrung die bessere Lösung, als wenn sich kritische Gruppen auf den unterschiedlichsten Wegen, von sozialen Medien über Presse bis hin zu Störaktionen, zu Wort melden, wenn bereits Entscheidungen getroffen wurden.

Ein weiterer wichtiger Aspekt historischer Gärten ist die Kleinräumigkeit im Vergleich zur umgebenden Landschaft. Damit verbunden sind häufig auch kleinere Streifgebietsoder Territoriumsgrößen im Vergleich zu Wald- oder Agrarökosystemen. Dadurch sowie durch eine gute Nahrungsbasis und oftmals das Fehlen bestimmter Mortalitätsfaktoren (insbesondere Bejagung) leben in historischen Gärten oft deutlich mehr Individuen pro Flächeneinheit als außerhalb. Während dies im Falle erwünschter Tiere positiv zu sehen ist, macht es den Umgang mit konfliktträchtigen Arten schwieriger.

\section{Direkte Eingriffe in die Bestände}

Will man das Anwachsen der Bestände begrenzen, steht in historischen Gärten nur ein vergleichsweise eingeschränktes Spektrum an Methoden zu Verfügung. Eine Bejagung ist aus rechtlichen, Sicherheits- oder politischen Gründen oftmals nicht oder nur in geringem Umfang möglich. Bei kleineren Tieren wie Wildkaninchen oder kleinen Raubsäugern stehen alternative Jagdmethoden in Gestalt etwa der Baujagd (Kaninchen, Fuchs) oder die Fangjagd mit Lebendfangfallen zur Verfügung. Eine Bejagung mit der Schusswaffe ist aus den erwähnten Gründen oft schwieg. Sicherheits- und Akzeptanzgründe sind das größte 
Problem. Ob hier die Jagd mit Pfeil und Bogen hilfreich ist, wäre zu untersuchen. Für das Schwarzwild kommen theoretisch noch sogenannte Frischlingsfänge in den Händen sehr erfahrener Fachleute in Frage. Diese Methode ist allerdings nicht in allen Bundesländern zulässig und aus Tierschutzgründen hochgradig umstritten (vgl. Herzog 2019).

Unter Laien herrscht nicht selten die Vorstellung, dass unerwünschte Tiere eingefangen und umgesiedelt werden können. Unlängst erfolgte etwa eine Umsiedlungsaktion der Biber aus dem Schlosspark Sanssouci (o. Verf. 2018). Leider ist die Umsiedlung nur in den seltensten Fällen eine nachhaltige Lösung. Zum einen scheitert die Maßnahme oft an rechtlichen Regelungen insofern, als das Aussetzen von Wildtieren in Mitteleuropa strengen gesetzlichen Regeln unterliegt. Das gilt natürlich auch für eingefangene Tiere, die anderweitig wieder ausgesetzt werden sollen. Darüber hinaus bedarf es geeigneter, hinreichend weit entfernter Lebensräume für die Ansiedlung entnommener Tiere. Bei mobilen Arten besteht sonst immer das Risiko, dass sie in ihre angestammten Habitate beziehungsweise Territorien zurückkehren.

Der häufig geäußerte Wunsch, man möge die Tiere doch einem Tierpark oder zoologischen Garten zuführen, ist aus unterschiedlichen Gründen meist nicht zu verwirklichen. Einerseits ist es aus Sicht des Tierwohls äußerst fragwürdig, Tiere, die in Freiheit aufgewachsen sind, als erwachsene Tiere einzusperren. Zum anderen ist die Kapazität und auch die Bereitschaft, bestimmte Arten wie etwa Waschbären oder Marder aufzunehmen, ausgesprochen begrenzt.

\section{Vergrämung}

Will oder darf man Tiere, die in historischen Gärten Probleme verursachen, nicht töten oder verfrachten, besteht grundsätzlich die Möglichkeit, sie zu vergrämen, d.h. sie durch die Anwendung unangenehmer Reize und Erfahrungen so zu beeinflussen, dass sie ein bestimmtes Gebiet in Zukunft meiden. Das Grundproblem bei der Vergrämung besteht darin, dass solche unangenehmen Erfahrungen regelmäßig wiederholt werden müssen und dass sie genau dadurch mit der Zeit an Wirksamkeit verlieren. Die Tiere lernen, das unangenehme Agens entweder zu tolerieren (etwa bei olfaktorisch oder akustisch aktiven Agentien) oder zu vermeiden (indem sie etwa über Elektrozäune springen).

Methodisch in eine ähnliche Richtung geht die Vorstellung, durch eine unattraktive Gestaltung des Lebensraumes die Siedlungsdichte bestimmter Tiere zu reduzieren. In historischen Gärten ist der Lebensraum allerdings meist vorgegeben, sodass hier nur wenige Handlungsoptionen bestehen.

Ein großräumigeres Problem stellen heute die begrünten Achsen vieler Städte dar, die nicht nur die (erwünschte) Durchlässigkeit der Landschaft als solche fördern, sondern zugleich auch den weniger erwünschten Arten, etwa dem Schwarzwild, die Besiedlung der Stadt erleichtern. Hier sind also immer Kompromisse erforderlich, eine eindeutig »richtige« Vorgehensweise gibt es nicht. 


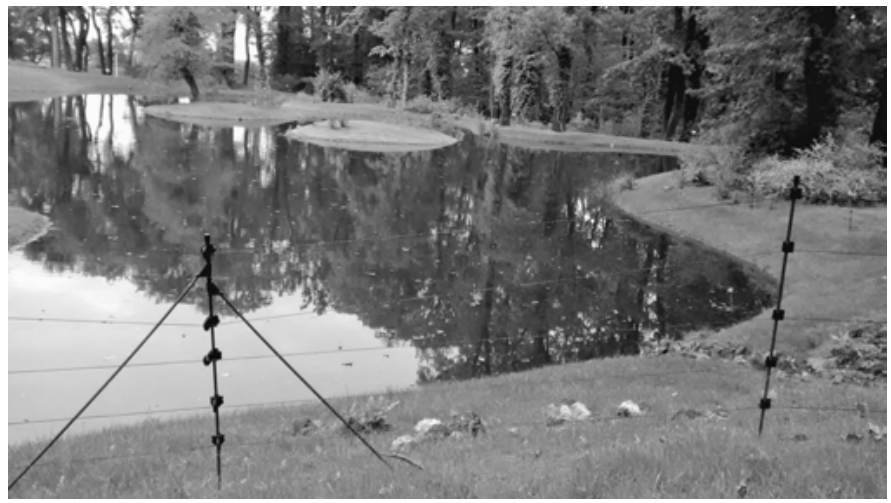

4 Elektrozäune gegen das Schwarzwild (Sus scrofa) wie hier in Babelsberg verursachen hohe Kosten, sind ästhetisch ungünstig und langfristig kaum wirksam.

\section{Repellente Maßnahmen und Zäune}

Repellente, also unmittelbar durch abschreckende optische, olfaktorische oder akustische Reize wirkende Maßnahmen, sind eher kurzfristig wirksam. Daher wird zunehmend darüber nachgedacht, historische Gärten oder Teile davon durch Zäune gegen Wildtiere zu sichern, was durchaus erfolgreich sein kann, allerdings dem Landschaftsbild oft abträglich ist oder (falls die Zäunung großräumig erfolgt) wiederum unerwünschte Zerschneidungseffekte der Landschaft mit sich bringt (Abb. 4).

Wenn Biber durch Dämme oder Biberburgen eine Überflutungsgefahr provozieren, können diese Bauwerke in Ausnahmefällen auch aktiv zerstört werden. Allerdings kann es passieren, dass die Biber an der gleichen Stelle mit einem Neubau beginnen.

Bei Gebäuden wird, wenn etwa Steinmarder, Waschbären oder Tauben sie besiedeln, nicht selten versucht, Zugänge zu Dachboden oder anderen Gebäudeteilen durch bauliche Maßnahmen zu versperren. Auch diese Maßnahme ist gelegentlich erfolgreich, hat allerdings den Nachteil, dass auch andere - erwünschte - Arten wie Turmfalke (Falco tinnunculus), Schleiereule (Tyto alba) oder Fledermäuse Lebensraum verlieren.

\section{Historische Gärten als Prüfstein für ein hochentwickeltes Wildtiermanagement}

Der vorliegende Beitrag zeigt die doch recht komplexe Situation der Wildtiere in historischen Gärten auf. Tiere können aus Sicht des Betreibers eines historischen Gartens etwa Bestandteil der Garteninstallation sein, sie können aus ästhetischen oder umweltpädagogischen Gründen erwünscht sein, sie können allerdings auch wegen der von ihnen verursachten Schäden an Gebäuden und Gartenanlagen ausgesprochen unerwünscht sein.

Diese noch vergleichsweise klare Struktur verschwimmt, wenn wir zusätzlich die Ansprüche und Einstellungen der Gartenbesucher, nicht selten aus einer sehr heterogenen und 
in Bezug auf natürliche Ressourcen sehr spezifisch sozialisierten urbanen Bevölkerungsgruppe stammend, betrachten. Dementsprechend verwundert es nicht, dass für historische Gärten derzeit keine Routinelösungen für den Umgang mit Menschen und Tieren existieren.

Ein typisches Beispiel ist das Auftreten von Wildschweinen in historischen Gärten. Diese Tiere sind aus der Perspektive des Grundeigentümers meist ausgesprochen unerwünscht, die intensive Reduktion des Bestandes wird erwartet. Die Besucher eines historischen Gartens wiederum haben andere Interessen. Sie sind den Tieren gegenüber entweder indifferent eingestellt oder von ihnen fasziniert, füttern sie und genießen sie als ein hochemotionales Naturerlebnis. Andere Menschen ängstigen sich und meiden entsprechende Flächen, auf denen sie mit Wildschweinen rechnen. Die letztere Gruppe ist allerdings ihrerseits inhomogen hinsichtlich der Frage, ob die Tiere regelmäßig getötet (also etwa bejagt) werden sollen oder ob sie etwa eingefangen und anderweitig verfrachtet oder kontrazeptiv behandelt werden sollen (oft geäußerte Vorstellungen; beides aus fachlicher Sicht ausgesprochen unrealistische Ansätze). Ein Managementkonzept für Wildtiere in historischen Gärten, soviel zeigt bereits dieses einfache Beispiel, muss alle betroffenen Interessengruppen und ihre Ziele und Ansprüche mit einbeziehen. Nur durch intensive Kommunikation im Vorfeld mit allen betroffenen Gruppen und eine wissenschaftliche Begleitung sämtlicher Maßnahmen werden wir hierbei zu tragfähigen Kompromisslösungen finden. Die allerdings können in Zukunft beispielhaft nicht nur für den Umgang mit Wildtieren in urbanen Räumen, sondern in unserer offenen und zunehmend durch ein »Grundmisstrauen« der Menschen geprägten Gesellschaft insgesamt sein.

\section{Literaturverzeichnis}

Bateman, Philip W.; Fleming, Patricia A. (2012): Big city life: carnivores in urban environments. In: Journal of Zoology 287, 1-23.

Berthold, Peter (1976): Animalische und vegetabilische Ernährung omnivorer Singvogelarten: Nahrungsbevorzugung, Jahresperiodik der Nahrungswahl, physiologische und ökologische Bedeutung. In: Journal für Ornithologie 117, 145-209.

DeCandido, Robert; Allen, Deborah (2006). Nocturnal hunting by Peregrine Falcons at the Empire State Building, New York City. In: The Wilson Journal of Ornithology 118, 53-58. https://doi.org/10.1676/15594491(2006)118[0053:NHBPFA]2.0.CO;2 (14.06.2018).

Ditchkoff, Stephen S.; Saalfeld, Sarah T.; Gibson, Charles J. (2006): Animal behavior in urban ecosystems: modifications due to human-induced stress. In: Urban Ecosystems 9.1, 5-12.

Dominoni, Davide M.; Greif, Stefan; Nemeth, Erwin; Brumm, Henrik (2016): Airport noise predicts song timing of European birds. In: Ecology and Evolution 6.17, 6151-6159.

Drewitt, Edward J.A.; Dixon, Nick (2008). Diet and prey selection of urban-dwelling Peregrine Falcons in southwest England. In: British Birds 101, 58-67.

Geist, Valerius (2007): How close is too close? Wildlife professionals grapple with habituating wildlife. In: The Wildlife Professional 1, 34-37.

Hackländer, Klaus; Schneider, Susanne; Lanz, Johann D. (2014): Einfluss von Hauskatzen auf die heimische Fauna und mögliche Managementmaßnahmen. Gutachten, Universität für Bodenkultur, Wien. https:// www.dib.boku.ac.at/fileadmin/data/H03000/H83000/H83200/Publikationen/KH_Gutachten_Hauskatze _Feb2014.pdf (14.06.2018).

Herzog, Sven (2019): Wildtiermanagement. Wiebelsheim: Quelle \& Mayer. 
Hope, Jack (2000): The geese that came in from the wild. In: Audubon 102, 122-126.

Longcore, Travis; Rich, Catherine (2004): Ecological light pollution. In: Frontiers in Ecology and the Environment 2.4, 191-198.

MacDonald, Sheila M.; Mason, Christopher F. (1994): Status and conservation needs of the otter (Lutra lutra) in the western Palearctic. Strasbourg: Council of Europe Press.

McDonald, Jennifer L.; Maclean, Mairead; Evans, Matthew R.; Hodgson, Dave J. (2015): Reconciling actual and perceived rates of predation by domestic cats. In: Ecology and Evolution 5.14, 2745-2753.

McKinney, Michael L. (2002): Urbanization, biodiversity, and conservation. In: Bioscience 52, 883-890.

McKinney, Michael L. (2006): Urbanization, as a major cause of biotic homogenization. In: Biological Conservation $127,247-260$.

O. Verf. (2018): Schlosspark Sanssouci: Sechs Biber gefangen und umgesiedelt. https://www.berlin.de/aktuelles/ berlin/5063604-958092-schlosspark-sanssouci-sechs-biber-gefang.html (14.06.2018).

Partecke, Jesko; Schwabl, Ingrid; Gwinner, Eberhard (2006): Stress and the city: urbanization and ist effects on the stress physiology in European blackbirds. In: Ecology 87.8, 1945-1952.

Plumer, Liivi; Davison, John; Saarma, Urmas (2014): Rapid Urbanization of Red Foxes in Estonia: Distribution, Behaviour, Attacks on Domestic Animals, and Health-Risks Related to Zoonotic Diseases. In: PloS ONE 9.12. e115124.doi:10.1371/journal.pone.0115124 (14.06.2018).

Ring, Caroline; Jeske, Ann-Kathrin (2017): Im Tiergarten leben jetzt neue Krebse - und alarmieren Forscher. In: Welt 23.08.2017. https://www.welt.de/wissenschaft/article167946948/Im-Tiergarten-leben-jetzt-neueKrebse-und-alarmieren-Forscher.html (14.06.2018).

Stein, Bruce A.; Kutner, Lynn S.; Adams, Jonathan S. (2002): Precious heritage: the status of biodiversity in the United States. Oxford: Oxford University Press.

\section{Bildnachweis}

1-3 Sven Herzog; ; 4 Insa Christina Herzog. 